\title{
Constraints on pre-main-sequence evolution from stellar pulsations
}

\author{
M. P. Casey ${ }^{1}$, K. Zwintz ${ }^{2}$ and D. B. Guenther ${ }^{1}$ \\ ${ }^{1}$ Institute for Computational Astrophysics, Department of Astronomy and Physics, \\ Saint Mary's University, Halifax, NS B3H 3C3, Canada \\ email: mcasey@ap.smu.ca, guenther@ap.smu.ca \\ ${ }^{2}$ Institute of Astronomy, University Leuven, Leuven, Belgium \\ email: konstanze@ster.kuleuven.be
}

\begin{abstract}
Pulsating pre-main-sequence (PMS) stars afford the earliest opportunity in the lifetime of a star to which the concepts of asteroseismology can be applied. PMS stars should be structurally simpler than their evolved counterparts, thus (hopefully!) making any asteroseismic analysis relatively easier. Unfortunately, this isn't necessarily the case. The majority of these stars (around 80) are $\delta$ Scuti pulsators, with a couple of $\gamma$ Doradus, $\gamma$ Doradus $-\delta$ Scuti hybrids, and slowly pulsating B stars thrown into the mix. The majority of these stars have only been discovered within the last ten years, with the community still uncovering the richness of phenomena associated with these stars, many of which defy traditional asteroseismic analysis.

A systematic asteroseismic analysis of all of the $\delta$ Scuti PMS stars was performed in order to get a better handle on the properties of these stars as a group. Some strange results have been found, including one star pulsating up to the theoretical acoustic cut-off frequency of the star, and a number of stars in which the most basic asteroseismic analysis suggests problems with the stars' positions in the Hertzsprung-Russell diagram. From this we get an idea of the constraints - or lack thereof - that these results can put on PMS stellar evolution.
\end{abstract}

Keywords. stars: pre-main-sequence, stars: oscillations, stars: fundamental parameters

\section{Introduction}

Pre-main-sequence (PMS) stars afford the earliest opportunity in the lifetime of a star to apply the techniques of asteroseismology, and therefore the earliest test of stellarevolution models. PMS stars are stars that have only recently emerged from the cloud from which they were formed, and have yet to start burning hydrogen into helium in their cores. Their primary source of energy is the conversion of gravitational potential energy into light; hence they are slowly contracting to the zero-age main-sequence (ZAMS) of hydrogen burning. Stars with mass between about 1.5 and $5 M_{\odot}$ can be shown to spend part of their evolution in the classical instability strip of the Hertzsprung-Russell diagram (HRD), and might be expected to pulsate. However, most of the discoveries have come within the past ten years, and the total number of confirmed or candidate stars is only around 80 objects, with the vast majority being $\delta$ Scuti stars, with a couple of $\gamma$ Doradus stars and $\delta$ Scuti- $\gamma$ Doradus hybrids thrown into the mix. There are also a couple of slowly pulsating B-star candidates. These proceedings will focus on the $\delta$ Scuti stars, but please see K. Zwintz's contribution, also in this volume, for the remainder. The study of this class of star is quite a new field!

Like their more-evolved counterparts, PMS $\delta$ Scuti stars are generally A stars, pulsating in pressure modes at frequencies ranging from approximately 5 to $80 \mathrm{~d}^{-1}$, or 55 to $925 \mu \mathrm{Hz}$, corresponding to periods of around 18 minutes to 5 hours. So far, it has been found that all confirmed or candidate members of the class are low-amplitude $\delta$ Scuti 


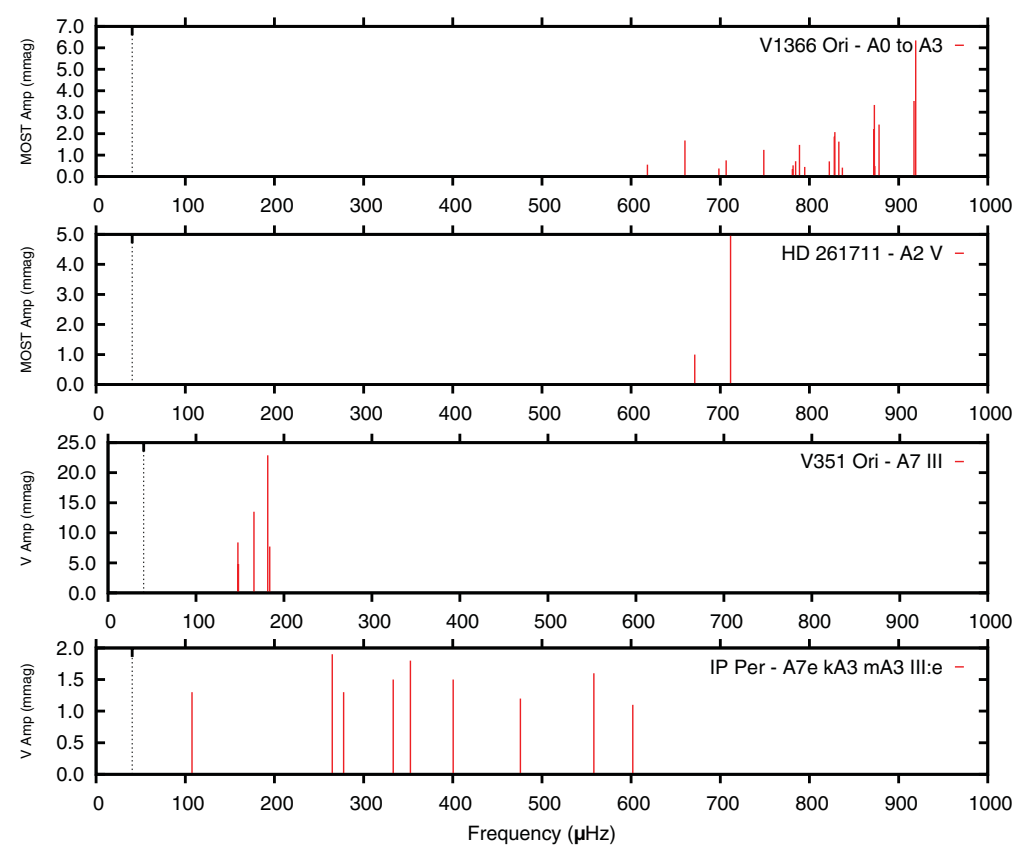

Figure 1. Sample PMS pulsation spectra. Sources of spectra: V1366 Ori, Casey et al. (2013); HD 261711, Zwintz et al. (2009b); V351 Ori, Ripepi et al. (2003); IP Per, Ripepi et al. (2006).

stars, with pulsation amplitudes in the millimagnitude range, typically simultaneously pulsating in many independent frequencies at once, both in radial and non-radial modes. Figure 1 shows four sample pulsation spectra.

\subsection{A brief history}

The first discovery of PMS $\delta$-Scuti candidates was of V588 and V589 Mon in the young open cluster NGC 2264 by Breger (1972). In this cluster only the O and B stars are on the main sequence, hence these two A stars (if cluster members) would be PMS stars less than 10 million years of age. Despite this encouraging start, it was $20+$ years later before the next member of the class was discovered, V856 Sco (HR 5999) by Kurtz \& Marang (1995).

As an Herbig Ae (HAe) star in the Lupus III dark cloud, the discovery of pulsations in V856 Sco partially revealed why discoveries of PMS were so scarce up to this point in time. Herbig Ae stars are thought to be young stars still surrounded by circumstellar material left over from the star-formation process. This circumstellar material carries a number of consequences: 1) It manifests itself in the hydrogen Balmer lines as emission; hence the $A e$ designation for the star. 2) It causes an infrared excess in the spectral energy distribution of the star (e.g. see Figure 8 of van den Ancker et al. 1996), which makes the intrinsic properties of the star harder to ascertain. 3) If the circumstellar material is clumpy, aperiodic and unpredictable eclipses of the light from the star of up to 2.5 magnitudes in depth, will result (a UX Ori variable). The depth of each "eclipse" can vary considerably from one extinction event to the next. Fortunately, the time scale for events is on the order of days to weeks, so with the right cadence of observations, UX Ori variability can be readily distinguished from $\delta$ Scuti variability. However, it is unlikely that $\delta$ Scuti variability will be discovered atop the UX Ori variability unless the $\delta$ Scuti variability is specifically sought. 4) The observed colour of a UX Ori variable changes as an eclipse event deepens, becoming redder and redder with increased 
extinction until the depth reaches 1.5 to 2 magnitudes in $V$, at which point "blueing" might start to occur - the colour of the star becomes bluer with increased extinction as the observations become contaminated with reflected blue light from the circumstellar material (again, see van den Ancker et al. 1996). The above four points make the intrinsic properties of an HAe star particularly hard to determine, and any $\delta$ Scuti variability hard to spot compared to (say) a more evolved A star. It is quite easy to misplace an HAe star in the HRD; when this happens even the most basic asteroseismic analysis of the star will fail, as will be demonstrated below.

\section{Theory}

The first theoretical asteroseismic analysis of a PMS $\delta$ Scuti star was that of V856 Sco by Marconi \& Palla (1998), in which an instability strip was calculated for the first three radial orders of pulsation. The calculated strip was roughly coincident with the instability strip of more-evolved counterparts. By the year 2000 a few more stars had been discovered; however it was only with Zwintz (2005) and the discovery of a number of PMS $\delta$ Scuti stars in open clusters that this subclass of pulsating star was fully established. Zwintz (2008) then contained a list of all known PMS confirmed or candidate $\delta$ Scuti stars to that date, and showed observationally that the PMS $\delta$ Scutis occupy the same part of a colour-magnitude diagram as their more-evolved counterparts. Additionally, by comparing observed to modelled frequencies of NGC 6383 170, Zwintz et al. (2007) conclusively showed for the first time a PMS $\delta$ Scuti star pulsating in non-radial modes. Guenther et al. (2007) further successfully fit 5 of 6 observed frequencies to models for NGC 653053,6 of 7 frequencies for star 13, and 9 of 9 frequencies of star 38, suggesting perhaps that the asteroseismic analysis of PMS $\delta$ Scuti stars might not be too difficult!

However, up to this point in time, all measurements had been with ground-based instruments, and so the advent of high-quality spaced-based observations from the $M i$ crovariablity and Oscillations of Stars (MOST) satellite mission (Walker et al. 2003) proved problematic; suddenly the observations had caught up the theory, and the theory was shown to be wanting. MOST observations of V1026 Sco (HD 142666) discovered 12 frequencies, of which only five could be fit to models at any one time (Zwintz et al. 2009a). Furthermore, MOST observations of the candidate PMS pulsators in NGC 2264 produced results that could sometimes be successfully compared to models, sometimes not (Guenther et al. 2009). It was now time for a systematic survey of the collective characteristics of PMS pulsators, e.g. pulsation frequencies (amplitudes, numbers), galactic environment, rotational velocities, etc., and to subject each star to as thorough an asteroseismic analysis as possible. The results of this study can be found in Casey (2011), with the highlights shown below.

\section{Results of a systematic study}

Collectively, the pulsation spectra of PMS $\delta$ Scuti stars reveal many phenomena: some pulsating over a wide range of frequencies that must span many different radial orders of pulsation; some pulsating in a group of low frequencies; some pulsating in a group of high frequencies. The problems associated with an asteroseismic analysis of many of these stars are demonstrated by the case of V1026 Sco (HD 142666), with twelve frequencies detected by MOST ranging from $66.8 \mu \mathrm{Hz}$ to $324.4 \mu \mathrm{Hz}$, including three frequencies, $f_{5}, f_{9}$, and $f_{12}$ that could possibly be a rotationally-split triplet. The best fit to the asteroseismic data (see Figs. 2 and 3), as bad as it is, is nowhere close to the position in the HRD as determined by other means (classification spectra, distance to star, etc.) One or the other or both must be wrong. How do we determine which might be the case? 

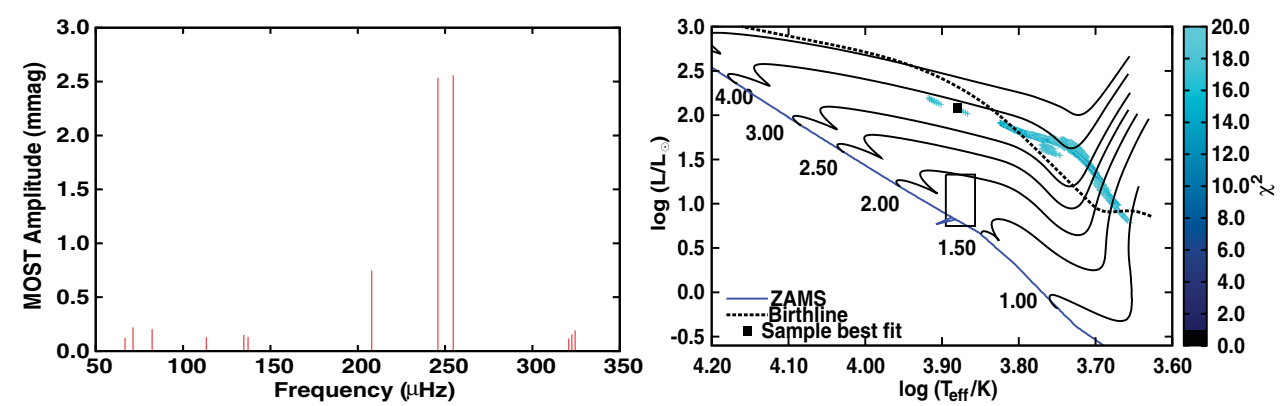

Figure 2. Left: V1026 Sco pulsation spectrum. Right: HRD position, including $\chi^{2}$ best-fit values to models. The "birthline" in this (and subsequent) figures refers to the birthline of Palla \& Stahler (1990).
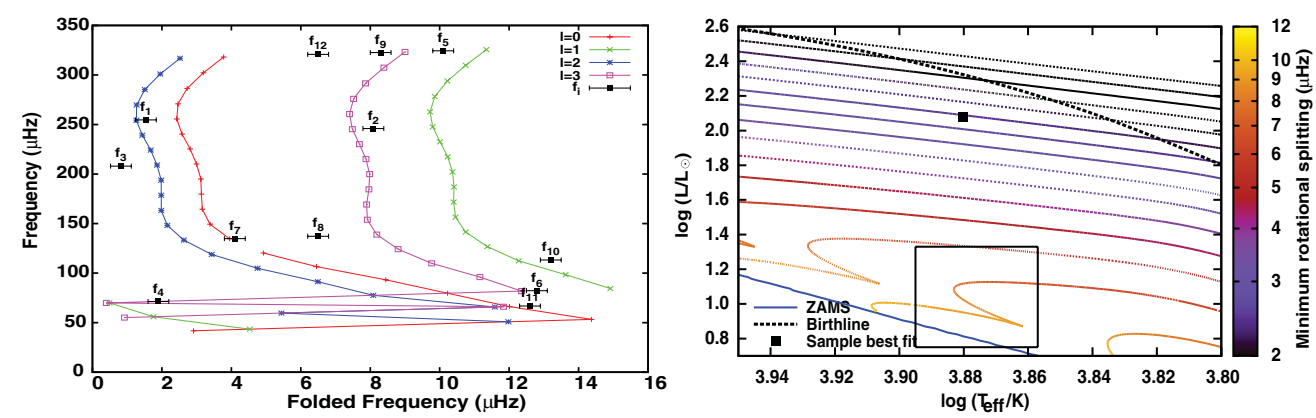

Figure 3. V1026 Sco echelle diagram (left) of best-fit solution (black dot at right), and estimate of expected minimum rotational splitting with $v \sin i=70 \mathrm{~km} \mathrm{~s}^{-1}$ (left).

\subsection{Rotational velocity}

A survey of the known $v \sin i$ values for PMS $\delta$ Scuti stars reveals that for the most part they rotate at relatively high velocities, generally $50 \mathrm{~km} \mathrm{~s}^{-1}$ or above, with 75 to $150 \mathrm{~km} \mathrm{~s}^{-1}$ being more typical (Casey 2011). As a star rotates, rotational splittings of non-radial $l$ modes appear (called multiplets) compared to a non-rotating model (the rotation breaks the spherical symmetry of the system). At low velocities the splittings are small and symmetric, and relatively easy to spot (if sufficient resolution is obtained in the frequency domain). However, if the velocity is large enough, the splittings become asymmetric, and multiplets of various non-radial $l$ orders can overlap, making it extremely difficult to identify any systematic spacings that may exist without some sort of statistical analysis (e.g. see Deupree \& Beslin 2010; Deupree 2011). In the case of V1026 Sco numerous $v \sin i$ values are reported, but the lowest value of $70 \pm 2 \mathrm{~km} \mathrm{~s}^{-1}$ is reported by Mora et al. (2001). Figure 3 shows an approximate estimate of the minimum rotational splitting one might expect for the star given this value for $v \sin i$. For the HRD position given by the box, we would expect splittings of around 10 to $14 \mu \mathrm{Hz}$, ruling out the high-frequency triplet as members of one multiplet, but more likely to be a chance alignment of overlapping multiplets. However, for the region of the HRD from which the best-fit solution occurs (black square dot), minimum splittings of around $2 \mu \mathrm{Hz}$ are found, indicating the possibility that the triplet (or perhaps maybe two frequencies of the triplet) are members of one rotationally-split multiplet. If the latter is true, then the problem is to figure out why the non-asteroseismic HRD position is so different; perhaps the distance to the star is wrong, or the extinction has been underestimated, either of which might significantly underestimate the luminosity of the star. Regardless, rotation 

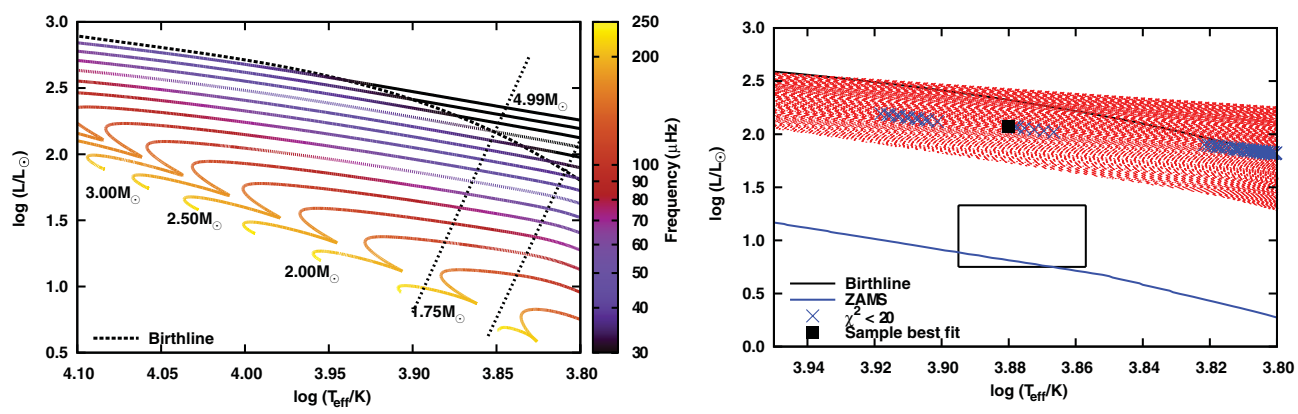

Figure 4. Left: fundamental frequencies of PMS models. Right: models with the fundamental frequency $<f_{11}$ of V1026 Sco (shaded region), along with location of best-fit models to the observed pulsation spectrum.

seems to be the primary reason for which detailed asteroseismic analysis of PMS stars seems to fail. At least two-dimensional modelling that includes rotation, such as that by Deupree \& Beslin (2010), but specifically for PMS stars, is required for progress. However, in the interim there are other attributes of the pulsation spectra that allow us to gain some insight into these stars.

\subsection{Lowest pulsation frequency and the fundamental mode of pulsation}

If we assume all frequencies in a spectrum are pressure modes ( $p$-modes), then some interesting limits can be put on the luminosities of stars. This is not to say that these frequencies are $p$-modes, but if the assumption is made, what are the consequences of this assumption? In the case of V1026 Sco, the lowest-detected frequency is $f_{11}=66.8 \mu \mathrm{Hz}$, or $5.78 \mathrm{~d}^{-1}$. If this is a $p$-mode, then one would expect this frequency to be higher than the frequency of the fundamental mode of star. Therefore, one would eliminate any stellar model from consideration for which the fundamental frequency is higher than the star's lowest detected frequency. The result is shown in Fig. 4. Given that the fundamental frequency decreases with increasing luminosity, this eliminates all the lower-luminosity models that happen to be in agreement with V1026 Sco's non-asteroseismic position in the HRD, indicating that $f_{11}$ is not a $p$-mode, or the HRD position is wrong. More work is therefore needed on this star! Through this simple test, lower-luminosity constraints on 32 stars can be placed, with 10 stars found to be "out of position" in the HRD, that is, their asteroseismic and non-asteroseismic positions in the HRD are not in agreement.

\subsection{Highest pulsation frequency and the acoustic cut-off frequency}

In Casey et al. (2013) it was found that the HAe star V1366 Ori (HD 34282), also a $\delta$ Scuti star, is most likely pulsating at frequencies just below the theoretical acoustic cut-off frequency (ACF) of the star, a first for a $\delta$ Scuti star. The ACF is a theoretical maximum pulsation frequency above which a star should not be expected to pulsate (Aerts et al. 2010). If a signal above the ACF is detected, then an explanation is needed, such as the modified ACF that seems to exist in some roAp stars (e.g. Saio et al. 2012), or the convectively-driven pseudomodes that are observed in the Sun (Jiménez et al. 2011). Figure 5 shows that the ACF varies systematically in the HRD, decreasing with increased luminosity for a given effective temperature. Therefore, the highest pulsation frequency detected within a pulsation spectrum places maximum luminosity constraints on the star if the frequency should be high enough - the detected frequency needs to be below the ACF. In the case of V1026 Sco, with highest-detected frequency of $f_{5}=324 \mu \mathrm{Hz}$, this constraint eliminated some of the highest-luminosity models from 
Table 1. Stars determined to be out of position in the HRD according to asteroseismology. All stars are dimmer than asteroseismology would suggest. For V351 Ori it is the Hipparcos distance that causes trouble.

\begin{tabular}{ll}
\hline \multicolumn{1}{c}{ Star } & Star formation region or cluster \\
\hline 1) V375 Lac & LkH $\alpha$ 233 DC \\
2) V856 Sco & Lupus 3 DC \\
3) V1026 Sco & Sco R1 \\
4) PX Vul & Vul R2 \\
5) IP Per & Per OB2 \\
6) GSC 07380-01173 & NGC 6383 \\
7) WW Vul & In Vulpecula \\
8) VV Ser & Serpens Cloud \\
9) V351 Ori (Hip) & Orion B \\
10) CQ Tau & Taurus molecular cloud \\
\hline
\end{tabular}
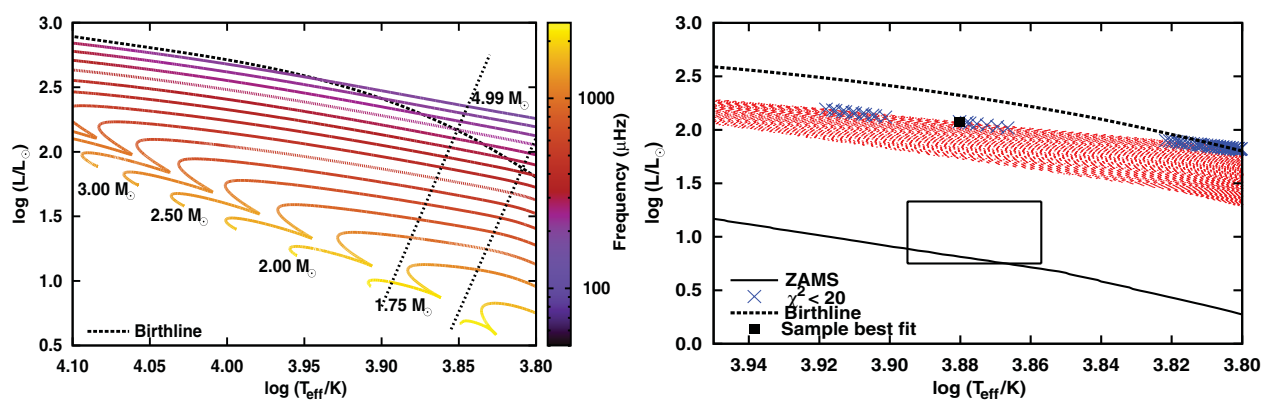

Figure 5. Left: ACFs of PMS models (models in the region just above the ZAMS with an ACF greater than $1000 \mu \mathrm{Hz}$ are not shown). Right: same as right side of Fig. 4, but also missing models with ACFs $>f_{5}$.

consideration, and it then becomes obvious in Fig. 5 why the best-fit models (as horrible as they are) were confined to a narrow horizontal band in the HRD: these are the only models under consideration that are able to even support the observed range of the pulsation spectrum. By using this test maximum-luminosity constraints can be placed on 20 of the candidate stars, although none of these constraints result in disagreement with the non-asteroseismic position of a star in the HRD.

\subsection{Out-of-position stars and HRD of PMS $\delta$ Scuti stars.}

Table 1 lists 10 candidate stars for which the asteroseismic and non-asteroseismic position in the HRD do not agree. Figure 6 contains the pulsation spectra of four stars and demonstrates how the limits work. A frequency falling within the right-hand-side shaded area puts a lower luminosity limit on the star via the fundamental-mode test - the lower the frequency, the higher the luminosity of the star must be for the frequency to be a $p$ mode. Similarly, a frequency falling within the left-hand-side shaded area puts an upper luminosity limit on the star via the ACF test - the higher the frequency, the lower the luminosity of the star must be for the frequency to be $p$-mode in a regular-atmosphere star (e.g. not a roAp star). For $\delta$ Scuti frequency ranges, there is only a very narrow frequency range between about 225 and $250 \mu \mathrm{Hz}$ into which a frequency can fall and not put a luminosity constraint on the star. 

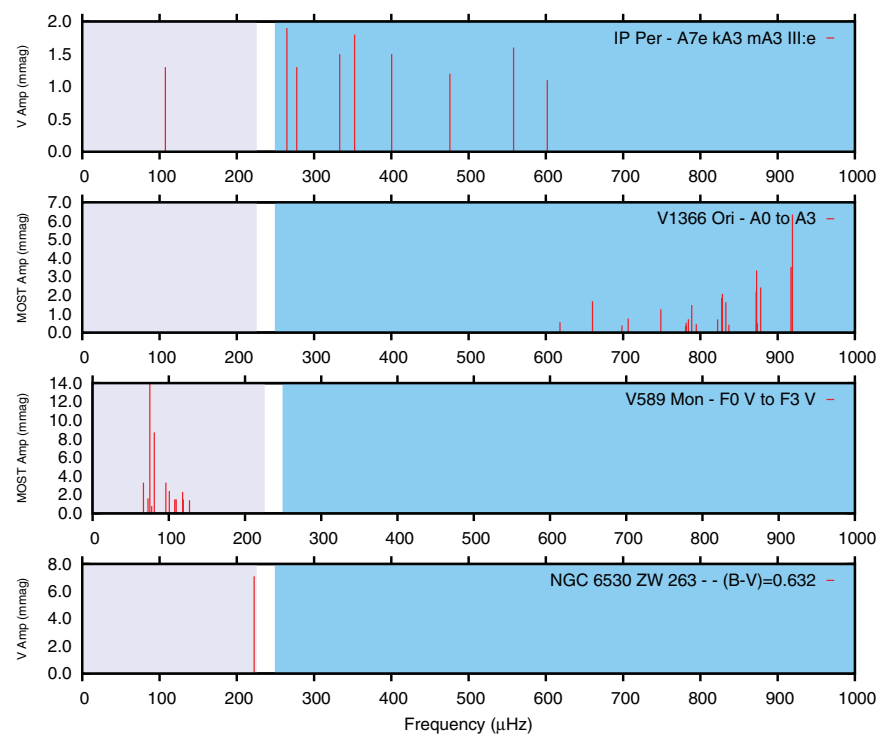

Figure 6. Limits on pulsation spectra: left shaded area, limits imposed by the fundamental frequency; right shaded area, limits imposed by the acoustic cut-off frequency. Sources of spectra: IP Per, Ripepi et al. (2006), V1366 Ori, Casey et al. (2013); V589 Mon, Guenther et al. (2009); NGC 6530 ZW 263, Zwintz \& Weiss (2006).
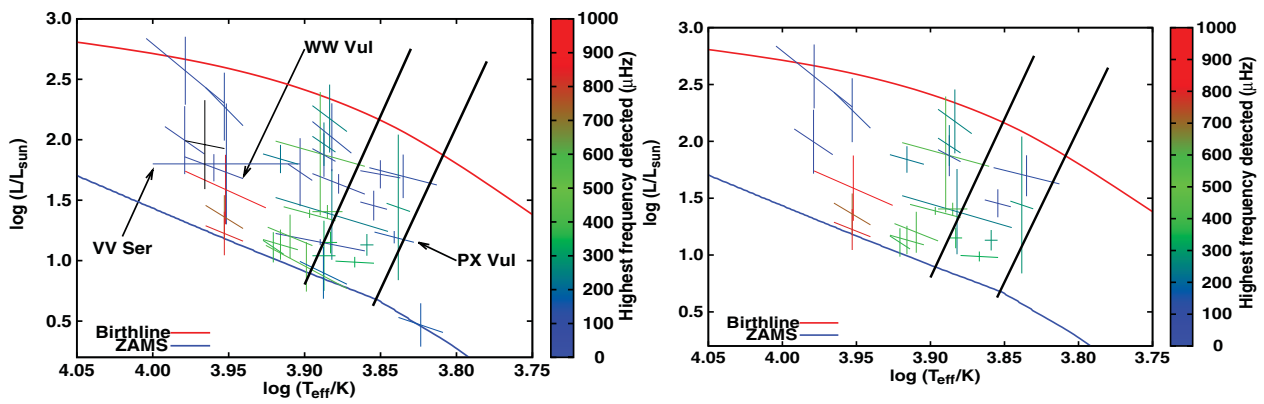

Figure 7. Left: HRD of PMS pulsators for which fundamental parameters could be deduced. Right: same as left, but with stars "out of position" in the HRD removed.

\section{HRD of $\delta$ Scuti pulsators}

After all this analysis, the left-hand side of Fig. 7 shows an HRD of PMS $\delta$ Scuti stars, along with the Marconi and Palla instability strip. Also indicated is the highest frequency detected within the pulsation spectrum of each star. For comparison, the lefthand side of the figure shows the same information except the "out-of-position" have been removed. All of the higher-frequency stars are in the lower left of the distribution, the lower-frequency are stars nearer to the top of the distribution, and the mid-range stars are spread around in the middle to the lower right. The stars to the left of the theoretical instability strip tend to be pulsating in orders higher than the first three radial modes. It will be interesting to see if further detections follow this pattern.

Two of the eliminated candidate stars (for which pulsation detections were too preliminary), PX Vul and WW Vul, have since been determined by MOST observations not to be pulsating, thus showing the usefulness of the above tests for determining which stars need further observations or theoretical work. Additionally, the lowest frequencies 
of another out-of-position star, VV Ser, may be $g$-modes (see Ripepi et al. 2007; Casey 2011); the fundamental-frequency test tends to flag such candidates fairly readily as being out of position in the HRD, whereas the HRD position may be fine, but it is the asteroseismic interpretation of the star that might need further work.

\section{Summary}

The systematic analysis of PMS $\delta$ Scuti pulsators from around 70 members/candidates, with 40 analyzed in greater detail, found examples of stars pulsating at all possible radial $p$-mode orders from the fundamental frequency up to the acoustic cut-off frequency. Rapid rotation was found to be the most probable reason for the failure of the detailed asteroseismic analysis of candidate stars. Using the fundamental mode as a test, minimum luminosity constraints could be placed on 32 stars, whereas the ACF test put maximum luminosity constraints on 20 stars. Ten "problem stars" were found, in which the nonasteroseismic and asteroseismic positions of the stars in the HRD were not in agreement, and for which future work (in particular) should be directed. The results are a new HRD for PMS $\delta$ Scuti stars, showing a distinct pattern of pulsation above the first three radial orders of pulsation, as originally calculated by Marconi \& Palla (1998). As for specific constraints on PMS evolution from asteroseismology, until the models catch up, it is very difficult to impose anything but the wide constraints demonstrated here. Future work (better HRD positions in particular) are another requirement. See the work of Zwintz et al., also in this volume, for more details.

\section{References}

Aerts, C., Christensen-Dalsgaard, J., \& Kurtz, D. W. 2010, Asteroseismology (Berlin: Springer) Breger, M. 1972, ApJ, 171, 539

Casey, M. P. 2011, Ph.D. thesis, Saint Mary's University, Canada

Casey, M. P., Zwintz, K., Guenther, D. B., et al. 2013, MNRAS, 428, 2596

Deupree, R. G. 2011, ApJ, 742, 9

Deupree, R. G. \& Beslin, W. 2010, ApJ, 721, 1900

Guenther, D. B., Kallinger, T., Zwintz, K., Weiss, W. W., \& Tanner, J. 2007, ApJ, 671, 581

Guenther, D. B., Kallinger, T., \& Zwintz, K., et al. 2009, ApJ, 704, 1710

Jiménez, A., García, R. A., \& Pallé, P. L. 2011, ApJ, 743, 99

Kurtz, D. W. \& Marang, F. 1995, MNRAS, 276, 191

Marconi, M. \& Palla, F. 1998, ApJ, 507, L141

Mora, A., Merín, B., \& Solano, E., et al. 2001, A\&A, 378, 116

Palla, F. \& Stahler, S. W. 1990, ApJ, 360, L47

Ripepi, V., Marconi, M., Bernabei, S., et al. 2003, A\&A, 408, 1047

Ripepi, V., Bernabei, S., Marconi, M., et al. 2006, A\& $A, 449,335$

Ripepi, V., Bernabei, S., Marconi, M., et al. 2007, A\&SA, 462, 1023

Saio, H., Gruberbauer, M., Weiss, W. W., Matthews, J. M., \& Ryabchikova, T. 2012, MNRAS, 420,283

van den Ancker, M. E., The, P. S., \& de Winter, D. 1996, A\& A, 309, 809

Walker, G., Matthews, J. M., Kuschnig, R., et al., 2003, PASP, 115, 1023

Zwintz, K. 2005, Ph.D. thesis, Universität Wien, Austria

Zwintz, K. 2008, ApJ, 673, 1088

Zwintz, K. \& Weiss, W. W. 2006, A\&A, 457, 237

Zwintz, K. \& Guenther, D. B., Weiss W. W. 2007, ApJ, 655, 342

Zwintz, K., Kallinger, T., Guenther, D. B., et al. 2009a, A\&SA, 494, 1031

Zwintz, K., Hareter, M., Kuschnig, R., et al. 2009b, A\& A, 502, 239 